\title{
A Preliminary Study of the Fluid Mechanics of Liquid Penetrant Testing
}

\section{S. Deutsch}

\section{Center for Mechanical Engineering and Process Technology, National Bureau of Standards, Washington, DC 20234}

February 28, 1979

\begin{abstract}
Some aspects of the fluid mechanics of liquid penetrant testing are considered. Penetration is represented by surface tension driven flow into defects of small defect width to depth ratio. Defect width is chosen so that both gravitational and non-continuum effects may be ignored. Penetration time is found to follow a Rideal-Washburn relation, in which

$$
t \sim \frac{l^{2} \mu}{\gamma R \cos \theta}
$$

where $t$ is time, $l$ defect depth, $\mu$ the dynamic viscosity, $\gamma$ the surface tension, $R$ the defect width and $\theta$ the contact angle. The proportionality constant, however, is shown to be strongly dependent on defect geometry and penetrant application procedure. The effect of slight fluid elasticity is shown to be negligible.
\end{abstract}

Key words: Defect geometry; fluid elasticity; liquid penetrant testing; Rideal-Washburn equation; surface tension.

\section{Introduction}

Liquid penetrants are frequently employed to locate microscopic (surface-accessible) defects in solid, non-porous, materials. The basic technique involves $[1,2,3]^{1}$

(a) cleaning the material to be tested

(b) applying the penetrant

(c) removal of excess penetrant

(d) application of a developer (e.g., an absorbent coating)

(e) visual inspection, and

(f) post inspection cleaning.

Success of the process depends on the ability of surface tension forces to draw the liquid into surface-accessible defects. In this report the identification and quantification of the factors which affect the extent and rate of penetration (and the somewhat analogous problem of rate of development) are considered.

\section{Scope of the Investigation}

We consider some aspects of the continuum fluid mechanics of surface tension driven flows. We further restrict our flow situations to cases in which

(a) the minimum defect dimension is much larger than the intermolecular distance of the liquid,

\footnotetext{
${ }^{1}$ Numbers enclosed in square brackets identify references.
}

(b) the rate of penetration is independent of the orientation of the defect, and

(c) the surface tension is a function of the substances involved and temperature only.

Restrictions $a$ and $\mathrm{b}$ can be used to define a range of characteristic defect dimensions. For liquids composed of simple molecules, we may estimate molecular spacing [4] as

$$
\lambda \equiv 3.5 \times 10^{-10} \mathrm{~m}
$$

Happel and Brenner [5], report continuum flows, such as Poiseuille flow, persist for Knudsen numbers (ratio of molecular spacing to some characteristic flow dimension (say, $R$ ) as high as 0.01 . With consideration of eq (1) then, restriction a implies that

$$
R \geq 3.5 \times 10^{-2} \mathrm{~m}
$$

Restriction b governs the largest defect size of interest. It requires that surface tension effects based on the minimum defect size be much larger than gravitational effects based on a defect depth $(l)$. Mathematically

$$
\frac{\gamma}{R}>>\rho g l
$$

where $\gamma$ is the surface tension, $\rho$ is the density, and $g$ is the acceleration of gravity. For an air-water interface, interpreting " $>>$ " as "100X", and with $l=100 R$, this implies that 


$$
R \leq 27 \mu \mathrm{m}
$$

In what follows then we shall deal with defect sizes of roughly $10^{-2} \mu \mathrm{m}$ to $10^{2} \mu \mathrm{m}$. Gravitational and non-continuum effects are ignored. Note further that our interpretation of eq (3) requires

$$
\frac{R}{l}<<1
$$

Restriction c removes the possibility that the surface tension might vary in some manner with the flow. We shall indicate where this assumption, though employed, is probably inapplicable and more detailed investigation warranted.

\section{Fundamentals}

The salient features of surface tension driven flow into defects may be illustrated by flow through an open ended capillary tube, ${ }^{2}$ as shown in figure 1 . It is a straightforward exercise to show, for steady axisymmetric flow at finite Reynolds number, ${ }^{3}$ that in the limit as $R / l \rightarrow 0$, the equations of motion become to order 1 ,

$$
\mu\left(\frac{\partial^{2} u}{\partial r^{2}}+\frac{1}{r} \frac{\partial u}{\partial r}\right)=\frac{\partial p}{\partial z}
$$

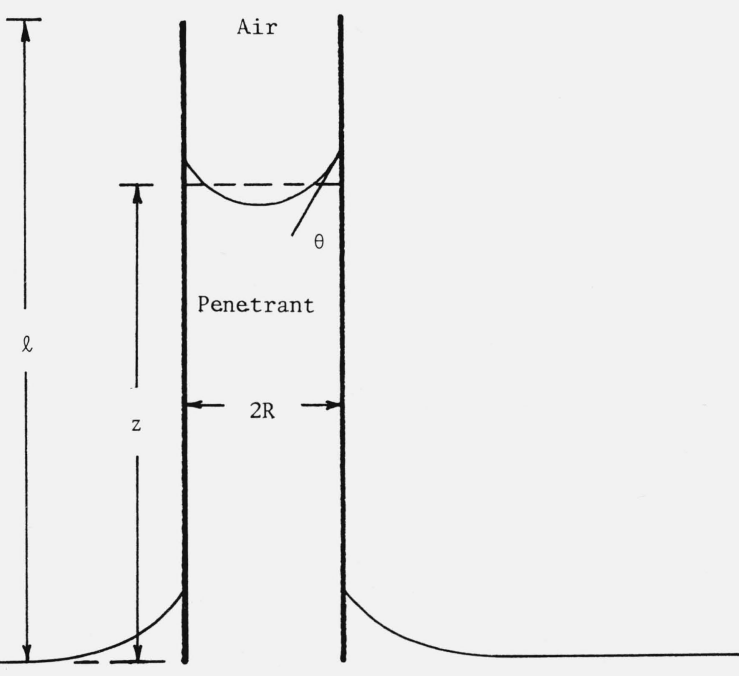

Figure 1. Capillary flow of penetrant in a small tube.

\footnotetext{
${ }^{2}$ That is, for $R / l \rightarrow 0$, we initially treat the closed downstream end of the defect as if it were at $t=\infty$, and had little effect. A more realistic approach, which accounts for the possibility of entrapped air is taken up in a later section.

${ }^{3}$ The same result could be reached by allowing $R e \equiv \bar{U} R / \nu \rightarrow 0$, where $\bar{U}$ is mean velocity and $\nu$ kinematic viscosity. However this is not generally as good an approximation as $R / l \rightarrow 0$ for the range of parameters considered here, and in view of equation 5 it is unnecessary.
}

Equation 6 is of course simply Poiseuille flow with solution

$$
u=\frac{G R^{2}}{4 \mu}\left(1-r^{2} / R^{2}\right)
$$

where

$$
G=-\frac{\partial p}{\partial z}
$$

In eqs (6), (7) and (8), $u$ is the axial velocity, $\mu$ the dynamic viscosity, $r$ the radial coordinate and $\partial p / \partial z$ the applied pressure gradient.

The volumetric flow rate can be found as

$$
Q=\pi G R^{4} / 8 \mu
$$

For a constant area tube $Q$ may be written as

$$
Q=\pi R^{2} d z / d t
$$

so that

$$
\frac{d z}{d t}=\frac{G R^{2}}{8 \mu}
$$

For capillary flows, the surface tension is related to the pressure jump across the meniscus by [4]

$$
\Delta p=\frac{2 \gamma \cos \theta}{R}
$$

where $\theta$ is the contact angle. Clearly then over a length $z$,

$$
G=\frac{\Delta p}{z}=\frac{2 \gamma \cos \theta}{R z}
$$

Using eqs (11) and (13)

$$
\frac{d z}{d t}=\frac{\gamma \cos \theta}{4 \mu} R / z
$$

or integrating and using $z=0$, at $t=0$ one finds for the penetration time, at $z=l$,

$$
t=\frac{2 l^{2} \mu}{R \cos \theta \gamma}
$$

Equation 15 is the Rideal-Washburn $[6,7]$ equation $^{4}$ and fundamentally identifies and relates the pertinent variables to the penetration time. Equation 15 has been experimentally confirmed by S. S. Kozlovskii [8] for liquid flow in glass tubes (with gravity) and within reasonable limits $(10 \%)$ by Good and Lin [9]. Kozlovskii [8] notes correctly that equation

${ }^{4}$ As applied to horizontal capillaries or equivalently following from the choice of defect sizes in the present case for negligible gravitational effects. 
15, derived from a steady flow assumption, should only be employed for times

$$
t>>R^{2} / \mu
$$

with $\rho \mathrm{R}^{2} / \mu$ being the viscous time scale. For the range of $R$ values considered here, however, eq (16) is not a serious limitation. Modifications of eq (15) to account for effects such as capillary narrowing and networks of capillaries are discussed in references [10-13]. If eq (15) is to be applied to a plug of solid powder (of interest in the development process) a tortuosity factor $(\xi)[14]$ must be introduced (i.e., $l \rightarrow \xi l)$. The factor $R / \xi^{2}$ may be evaluated experimentally.

\section{The Effect of Non-Circular Cross Section}

An obvious extension of the theoretical results of the previous section is to a more realistic representation of defects. To this end we consider flow through non-circular cross sections. The problem of steady state flow through straight "pipes" of uniform but non-circular cross section is highly developed. Under certain simplifying assumptions the problem reduces to one in potential flow theory and further is analogous to the problem of torsion of an elastic bar [15]. The development of the equations here closely follows Langlois [16].

Consider a flow, in the $\mathrm{x}_{3}$ direction of the form

$$
u_{3}=u\left(x_{1}, x_{2}\right) ; u_{1}, u_{2}=0
$$

being driven at steady state by a uniform pressure gradient.

Because of the form of eq (17) the nonlinear, inertia terms are identically zero, and continuity identically satisfied, so that the momentum equation becomes

$$
\Delta^{2} u+G / \mu=0 .
$$

Equation (18) is subject to

$$
u\left(x_{1}, x_{2}\right)=0
$$

on pipe periphery $\Gamma$. Here

$$
\Delta^{2}=\frac{\partial^{2}}{\partial x_{1}{ }^{2}}+\frac{\partial^{2}}{\partial x_{2}{ }^{2}}
$$

Equation (18) is of course a Poisson equation for the velocity. The system of eqs (18-20) may be shown to be equivalent to the Dirichlet problem ${ }^{5}$

$$
\Delta^{2} v=0
$$

with

$$
v=f\left(x_{1}, x_{2}\right) \text { on } \Gamma
$$

provided $f$ satisfies

$$
\Delta^{2} f=2
$$

Detailed solutions for some specific cross sections, as well as additional mathematical details may be found in Sokolnikoff [15] and Langlois [16].

We consider here the simple but illustrative case of an elliptical cross section. We adjust our coordinate system so that the most general ellipse can be written as

$$
\left(\frac{x_{1}}{a}\right)^{2}+\left(\frac{x_{2}}{b}\right)^{2}=1 \text {. }
$$

The solution of eqs (18-20) may be easily found to be [16]

$$
u=\frac{G}{2 \mu} \frac{a^{2} b^{2}}{a^{2}+b^{2}}\left(1-x_{1}^{2} / a^{2}-x_{2}^{2} / b^{2}\right)
$$

with volumetric flow rate

$$
Q=\frac{\pi G}{4 \mu} \frac{a^{3} b^{3}}{a^{2}+b^{2}} .
$$

Again $G$ may be estimated as

$$
G=\frac{\gamma \cos \theta}{s / b}\left(\frac{1}{a}+\frac{1}{b}\right)
$$

and penetration time is found to be, at $z=l$,

$$
t=\frac{2 l^{2} \mu}{\gamma \cos \theta} \frac{a^{2}+b^{2}}{(a+b) a b}
$$

The ratio of penetration times for elliptic to circular pipes of equivalent area may be found from eqs (15) and (28) to be

$$
\frac{t \text { ell. }}{t \text { cir. }}=\frac{\sqrt{a b}\left(a^{2}+b^{2}\right)}{a b(a+b)}
$$

Note that for $b=100 a$ say, penetration into the ellipse takes 9.9 times longer than into the equivalent area circle. Equation (29) is a reflection of the fact that a circular pipe produces more flow rate, at a given pressure gradient, than an elliptical one, at equivalent area.

\section{Entrapped Air}

It would seem possible, in applying penetrant, to trap air in some defects. The flow induced compression of this 
trapped air would, by reducing the effective pressure gradient, reduce flow rate and increase penetration time.

Let us consider the problem in which flow is developing slowly due to a continuously changing axial pressure gradient. It may be shown that the condition

$$
R / l \rightarrow 0
$$

is sufficient to guarantee slow axial development. By maintaining finite Reynolds number, the equations of motion become

$$
\mu\left(\frac{\partial^{2} u}{\partial r^{2}}+\frac{1}{r} \frac{\partial u}{\partial r}\right)=\left(P_{0}-P_{1}+\frac{2 \gamma \cos \theta}{R}\right) \frac{1}{l}
$$

Here $P_{0}$ is the atmospheric pressure and $P_{1}$ the pressure of the trapped air (initially atmospheric). Let us further take

$$
P_{1} V_{1}=C
$$

where

$$
C=\pi R^{2} l P_{0}
$$

The volume $V_{1}$ is then

$$
V_{1}=\pi R^{2}(l-z)
$$

so that

$$
P_{1}=\frac{P_{0} l}{(l-z)}
$$

The velocity may be found to be

$$
u=f(r) \frac{\left[2 \gamma \cos \theta(l-z)-P_{0} z R\right]}{l R(l-z)}
$$

with

$$
f(r)=\frac{R^{2}}{4 \mu}\left(1-r^{2} / R^{2}\right)
$$

Equations (36) and (37) clearly show that the flow develops slowly enough to retain the parabolic profile of Poiseuille flow while continuously adjusting its magnitude (and flow rate) in proportion to a constantly diminishing pressure gradient.

From eq (36) we note that the flow will cease when

$$
z / l=\frac{2 \gamma \cos \theta}{R P_{0}+2 \gamma \cos \theta}
$$

For $P_{0}$ of 1 atmosphere $\left(\sim 1 \times 10^{6}\right.$ dynes $\left./ \mathrm{cm}^{2}\right), R$ of $10^{2}$ $\mu \mathrm{m}$ and an air-water interface, eq (38) shows that the flow will cease at

$$
z / l \simeq 1.5 \%
$$

so that the effect can be quite dramatic; i.e., the penetrant will not penetrate $98.5 \%$ of the depth of the defect.

The flow rate, here a function of $z$, can be found directly as

$$
Q(z)=\frac{\left(2 \gamma \cos \theta(l-z)-P_{0} z R\right)}{l R(l-z)} \frac{\pi R^{4}}{8 \mu}
$$

Penetration time may be found, by integration of eq (40) to be

$$
\begin{aligned}
t=\frac{8 \mu l^{2}}{P_{0} R^{2}} & \left(\frac{1}{\left.\left(\frac{2 \gamma}{P_{0} R}\right) \cos \theta-1\right)^{2}}\right. \\
& \ln \left(\frac{2 \gamma}{P_{0} R} \cos \theta-\left(\frac{2 \gamma}{P_{0} R} \cos \theta-1\right) \frac{z}{l}\right. \\
& \left.\left.+\frac{\left.\left(\frac{2 \gamma}{P_{0} R} \cos \theta-1\right)\right)}{(l)}\right)\right)
\end{aligned}
$$

As one would anticipate, eq (41) shows that the time to reach a $z / l$ given by eq (38) is infinite. Both eqs (39) and (41) indicate that trapped air can have a large effect on the penetrant process.

It should be noted that unlike the case considered here, one major effect of the compression will probably be to alter the surface tension and/or change the shape of the interface. Additional research then, drawing on more detailed information on the physics of the interfacial region, may be fruitful.

\section{Effect of Fluid Elasticity}

This section is undertaken on the assumption that the addition of various dyes, fluorescents and emulsifiers might add a slight elasticity to the penetrating liquid. There is at present no experimental evidence to support (or refute) this hypothesis. However, as the effects of even slight elasticity on entrace type flows can be large [17], it would seem worthwhile to include at least the outline of an analysis. We should also note that perhaps "elasticizing" the fluid may prove to be useful in some aspects of the penetrant process (say in desensitizing the fluid to a certain defect size range).

For simplicity, consider steady axisymmetric fully developed pipe flow. For such a flow (termed viscometric), it is fair to state that the difference between a Newtonian and (slightly) viscoelastic fluid is that the latter will exhibit nonzero normal stresses. The creation of these extra normal stresses in entrance flows may lead to a large entry pressure drop [17, 18]. This extra pressure drop can substantially negate the applied pressure gradient. 
We consider a fluid in which the shear and normal stresses can be written as

$$
\tau^{r z}=\mu \frac{\partial u}{\partial r}
$$

and

$$
\tau^{\alpha \alpha}=C^{\alpha}\left(\frac{\partial u}{\partial r}\right)^{2 *}
$$

We further take

$$
C^{\alpha}=C^{\prime \alpha} \lambda \mu
$$

where $\lambda$ is a characteristic relaxation time. Equations (4244) are not unrealistic for weakly viscoelastic fluids in pipe flow.

Equation (42) taken together with more general equations of motion [18] permit a Poiseuille solution. Then

$$
\frac{\partial u}{\partial r}=\frac{-1}{2 \mu} r \frac{\partial p}{\partial z}
$$

so that

$$
\tau^{\alpha \alpha}=C^{\prime \alpha}\left(\frac{\lambda}{4 \mu} r^{2}\left(\frac{\partial P}{\partial z}\right)^{2}\right)
$$

Using eq (46), it is algebraically straightforward [18] to find as an expression for the effective pressure gradient

$$
\frac{-P_{e}}{z}=\frac{P_{a}}{z}+\frac{1}{8 \mu} C^{\prime \alpha}\left(\frac{R}{z^{3}}-P_{e}{ }^{2} \lambda\right) .
$$

Letting

$$
A=\frac{1}{8 \mu} C^{\prime \alpha} \frac{R^{2}}{z^{2}} \lambda
$$

one finds for the effective driving pressure

$$
P_{e}=\frac{-1 \pm \sqrt{1+4 A P_{a}}}{2 A}
$$

Note that for the penetrant problem

$$
P_{a}=\frac{2 \gamma \cos \theta}{R}
$$

If, as before, we take $R / l \rightarrow 0$, we note that $A \rightarrow 0$, for $z=$ $l$, and

\footnotetext{
* No sum intended on $\alpha$.
}

$$
P_{e} \rightarrow P_{a}
$$

The effect of elasticity then is apt to be small.

\section{Concluding Remarks}

Some aspects of the continuum fluid mechanics of penetrant flow have been examined. Characteristic defect sizes of $10^{-2}$ to $10^{2} \mu \mathrm{m}$ were considered. Gravitational and noncontinuum effects were neglected. The defect depth was everywhere assumed much larger than crack size.

Fundamentally, the penetrant process was found to obey a Rideal-Washburn $[6,7]$ type relation, so that penetration time

$$
t \sim \frac{l^{2} \mu}{R \gamma \cos \theta}
$$

Considerations of noncircular cross section and entrapped air, however, show that the proportionality constant in equation 52 is substantially geometry dependent and possibly also dependent on the penetrant application procedure. Elastic effects were shown to be negligible.

Geometric and process dependence, although somewhat expected, is certainly disquieting. Trapped air, for example, can essentially eliminate penetration ability over a certain range of defect sizes. Of course, some very fundamental questions such as what constitutes a sufficient penetration length, have not been considered. It would seem that a carefully controlled experimental investigation, coupled perhaps with computer simulations for complicated geometries, may be worthwhile.

\section{References}

[1] McMaster, R. C., NonDestructive Testing Handbook, 1, 6, The Ronald Press Company, N. Y., 1959

[2] Pasley, R. L., NonDestructive Testing, NASA SP-5113, 1973.

[3] Lomerson, F. O., Jr., Liquid Penetrants, Materials Evaluation, 36, 1 , 1978.

[4] Batchelor, G. K., An Introduction to Fluid Dynamics, Cambridge University Press, 1967.

[5] Happel, J. and Brenner, H., Low Reynolds Number Hydrodynamics, Prentice Hall, N. J., 1965.

[6] Washburn, E. W., Phys. Rev., 17, 273, 1921.

[7] Rideal, E. K., Phil. Mag., 44, 1152, 1922.

[8] Kozlovskii, S. S., Transactions of The Grozniy Petroleum Institute, No. 7, 1949. (In Russian); see Levich, V. G., Physicochemical Hydrodynamics, Prentice Hall, N. J., 1962.

[9] Good, R. J., Lin, N. J., Rate of Penetration of a Fluid into a Porous Body. II. Verification of the Generalization of the Washburn Equátion for Organic Liquids in Glass Capillaries, J. of Colloid \& Interface Science, 54, 1, 1976.

[10] Eley, D. D., and Pepper, D. C., A Dynamical Determination of Adhesion Tension, Trans. of the Faraday Soc., 42, 697, 1946.

[11] Perk, R. L., Jr. and McLean, D. A., Capillary Penetrance of Fibrous Materials, Industrial and Engineering Chemistry (Award Ed.), 6, 85, 1934. 
[12] Barren, R. M., Fluid Flow in Porous Media, Disc. Faraday Soc., 3, 61, 1948.

[13] Carman, P. C., Some Physical Aspects of Water Flow in Porous Media, Disc. Faraday Soc., 3, 72, 1948.

[14] Davies, J. T. and Rideal, E. K., Interfacial Phenomena, 2nd Edition, Academic Press, N. Y., 1963.

[15] Sokolnohoff, I. S., Mathematical Theory of Elasticity, McGraw-Hill, 1946.
[16] Langlois, W. E., Slow Viscous Flow, The Macmillan Company, N. Y.,

[17] Metzner, A. B., Uebler, E. A., and Chan Man Fong, C. F., Converging Flows of Viscoelastic Materials, AICHE Journal, 15, 750, 1969. [18] Darby, R., Viscoelastic Fluids, Marcel Dekkes Incorporated, New York, 1976. 